Received: 22 June 2017

Accepted: 26 October 2017

Published online: 13 November 2017

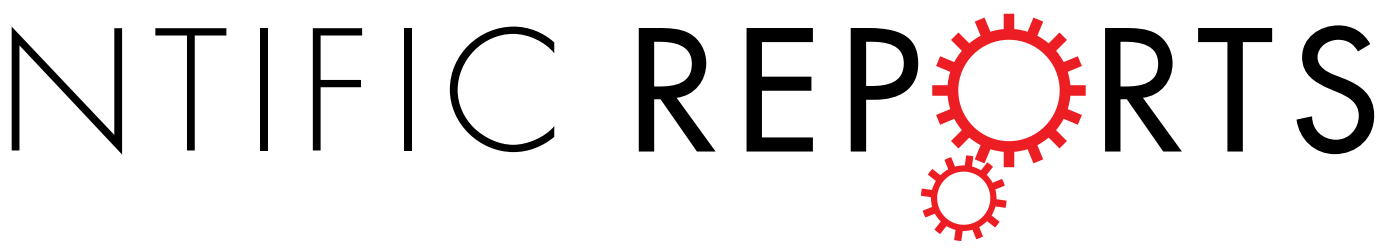

\title{
Microalbuminuria as a simple predictor of incident diabetes over 8 years in the Korean Genome and Epidemiology Study (KoGES)
}

Dong-Hyuk Jung ${ }^{1}$, Young-Sup Byun ${ }^{2}$, Yu-Jin Kwon ${ }^{1} \&$ Gwang-Sil Kim²

Microalbuminuria (MAU) is a common subclinical disease and related with cardiovascular outcome both in diabetic and non-diabetic patients. However, there is rare data about the effect of MAU on the development of diabetes. Thus, we aimed to investigate whether MAU is associated with the development of incident diabetes. A total of 3385 subjects without diabetes ( 1503 men and 1882 women; mean age, 53 years) who participated in the Ansung-Ansan cohort study from 2001-2002 (baseline) to 2011-2012 (fifth follow-up visit) were followed for a mean of 8 years. The prevalence of MAU at baseline was $10.8 \%$ (365 patients), and the incidence of newly developed diabetes during the follow-up period was $15.3 \%$ (56 patients) in subjects with MAU. The hazard ratio (HR) for development of diabetes was 1.43 (95\% confidence interval (CI) 1.07-1.91, p-value 0.016), independent of traditional risk factors for diabetes including pre-diabetes, age, obesity, and family history. The impact of MAU on diabetes was also significant in the non-pre-diabetic population (HR 2.08, 95\% Cl 1.07-4.03, p-value 0.031 ). In conclusion, our results show that incident MAU is associated with future development of diabetes and could be an early marker for diabetes, even in the non-prediabetic population.

Microalbuminuria (MAU) is a common subclinical disease whose prevalence ranges from $5-7 \%$ in the general population $^{1}$ and is about $30 \%$ in patients with hypertension $(\mathrm{HTN})^{2}$. MAU is an established predictor of micro and macrovascular complications in patients with type 2 diabetes. However, recent epidemiological and experimental data have indicated that MAU is an early marker of target organ damage and is associated with all-cause mortality, CVD incidence, and progression of chronic kidney disease in non-diabetic subjects ${ }^{3-5}$. In addition, several studies have reported that insulin resistance or prediabetes is associated with $\mathrm{MAU}^{6-8}$. However, few studies have explored the effect of MAU on the development of diabetes in the non-diabetic population.

We therefore aimed to investigate whether MAU is associated with the development of incident diabetes in the general Korean population using data from the Korea Genome and Epidemiology Study (KoGES).

\section{Research Design and Methods}

Study Population. Study subjects were individuals who participated in the Ansung-Ansan cohort study from 2001-2002 (baseline) to 2011-2012 (fifth follow-up visit). The Ansung-Ansan cohort study is an ongoing study that began in 2001 and involves biennial follow-up examinations. A total of 10038 patients were initially enrolled in the cohort. The design and baseline characteristics of the Ansung-Ansan cohort study have been previously published ${ }^{9,10}$. In this study, we included 4297 subjects whose urine albumin-to-creatinine ratio (UACR) was analysed at the first visit. From these 4297 subjects, we excluded 512 who had underlying diabetes and 400 who did not attend their follow-up examinations. Finally, a total of 3385 subjects without diabetes (1503 men and 1882 women; mean age, 53 years) were enrolled in the study. Subjects were followed for a mean of 8 years. Informed consent was obtained from all study subjects. The study protocol was approved by the ethics committee of the Korean Centre for Disease Control and the Yonsei University School of Medicine Institutional Review Board.

${ }^{1}$ Department of Family Medicine, Yongin Severance Hospital, Yonsei University College of Medicine, Yongin, Republic of Korea. ${ }^{2}$ Division of Cardiology, Department of Internal Medicine, Sanggye Paik Hospital Inje University College of Medicine, Seoul, Republic of Korea. Dong-Hyuk Jung and Young-Sup Byun contributed equally to this work. Correspondence and requests for materials should be addressed to G.-S.K. (email: zidan007@paik.ac.kr) 
and performed according to the principles of the Declaration of Helsinki. All data generated during this study are available.

Clinical and biochemical parameters. Study data included medical history, physical examination, information provided by a questionnaire, anthropometric measurements, and laboratory measurement. Information on medical history, family history, current use of medications, weekly alcohol consumption, and smoking status were obtained from all participants using a standard questionnaire.

Waist circumference was measured three times at the midpoint between the bottom of the ribcage and the top of the iliac crest using a fiberglass tape measure. Blood pressure was measured in the sitting position after 5 min of rest using a standard mercury sphygmomanometer.

Body mass index (BMI) was calculated as weight divided by height squared $\left(\mathrm{kg} / \mathrm{m}^{2}\right)$. Physical activity was classified into the following three categories: none, irregular ( $\leq 2$ episodes/week), and regular ( $\geq 3$ episodes/week) exercise. One episode of exercise was defined as exercising for at least $30 \mathrm{~min}$. The International Obesity Task Force (IOTF) and World Health Organization (WHO) regional office for the Western Pacific region recommend defining obesity in Asians as a BMI $\geq 25 \mathrm{~kg} / \mathrm{m}^{2}$. Subsequently, the Korean Society for the Study of Obesity (KSSO) adopted this definition. Thus, subjects were classified as "generally obese" if their BMI was $\geq 25 \mathrm{~kg} / \mathrm{m}^{2}$. Abdominal obesity was defined as a waist circumference $\geq 90 \mathrm{~cm}$ for male subjects and $\geq 85 \mathrm{~cm}$ for female subjects according to the criteria of the modified National Cholesterol Education Program's Adult Treatment Panel III (NCEP ATP III) Asian criteria for metabolic syndrome. Collected blood samples were delivered to and analysed at a central laboratory (Seoul Clinical Laboratories, Seoul, Korea). Plasma glucose, total cholesterol, triglycerides, and HDL cholesterol levels were determined using a Hitachi 747 chemistry analyser (Hitachi, Tokyo, Japan). The LDL cholesterol level was calculated using Friedewald's equation. The HbAlc level was measured by high-performance liquid chromatography on a Variant II instrument (BioRad Laboratories, Hercules, CA).

Definitions of MAU, diabetes, and prediabetes. MAU was defined as an UACR of $30-300 \mathrm{mg} / \mathrm{g}$. We excluded overt albuminuria, defined as UACR $>300 \mathrm{mg} / \mathrm{g}$. diabetes was defined as one of the following: (1) self-reported history of diabetes; (2) HbAlc level $\geq 6.5 \%$; or (3) use of an antidiabetic agent or insulin. Prediabetes was defined as a fasting plasma glucose level of 110 to $125 \mathrm{mg} / \mathrm{dl}$ or an $\mathrm{HbAlc}$ concentration of 5.7-6.4\%.

Statistical analyses. Data are expressed as mean and SD or as the number and percentage. Comparisons of baseline variables with respect to the presence or absence of MAU were analysed using Student's t test for continuous variables; categorical variables were analysed using the $\mathrm{x}^{2}$ test. We calculated the hazard ratios (HRs) for incident diabetes using Cox proportional hazards models with potential confounding variables. We included traditional risk factors for diabetes such as age, obesity, family history of diabetes, smoking status, physical activity and prediabetes in univariate analyses. Variables with $\mathrm{p}<0.15$ in the univariate analysis were entered into multivariate analysis. We developed two different models to estimate the risk of new onset diabetes. The basic model was based only on the parameters that were easy to assess without biochemical testing. The risk factors for the incidence of diabetes in the basic model were: age, family history of diabetes and obesity. Clinical model was created by adding MAU to the basic model. For each model, the area under a receiver operating characteristic curve (AROC) was calculated. Comparison of AUC curves was calculated using Delong method. This value represents an estimate of the probability that a model assigns a higher risk to those who develop diabetes within an 8-year follow up than to those who do not. All analyses were performed using SPSS Statistics for Windows version 18.0 (IBM, Armonk, NY). For all tests, a P value $<0.05$ was considered to indicate a statistically significant difference.

\section{Results}

The anthropometric and biochemical characteristics of the subjects are summarised according to the presence of MAU in Table 1. MAU was observed in 365 patients (10.8\%) at baseline, and the mean follow up duration was approximately 8 years. The development of diabetes was increased in subjects with MAU compared with subjects without MAU after 2 years of follow up; this gap widened over time (Fig. 1). The mean age, prevalence of hypertension, and level of $\mathrm{HbA1C}$ increased as baseline MAU category increased. Although there was no significant difference in the presence of metabolic syndrome, the proportions of subjects with high triglycerides and impaired fasting glucose were higher in the MAU group. There were no differences in activity or family history of diabetes. Of the 365 subjects with MAU, diabetes developed in 56 (15.3\%) during the 10-year follow-up period. This proportion was statistically significantly different than that in subjects without MAU. The incidences of composite cardiovascular disease and each component are shown in Table 2; no significant difference was observed according to the presence of MAU. Using a Cox proportional hazards model, we also investigated the clinical impact of MAU on the development of diabetes during the follow-up period. Participants with MAU had a higher risk for development of diabetes (hazard ratio [HR] 1.78, 95\% confidence interval [CI] 1.34-2.37, $\mathrm{p}$ value $<0.001$ ), as shown in Table 3. Moreover, this significant association remained after adjusting for other risk factors for diabetes including age, prediabetes, general and abdominal obesity, and family history. We next performed subgroup analysis. In the non-prediabetic group, the association was even greater (HR $2.08,95 \% \mathrm{CI}$ 1.07-4.03, $\mathrm{p}$ value 0.031 ), even after adjusting for confounding factors. The association between MAU and diabetes was more prominent in younger patients and in nonobese patients (Fig. 2). The AROC for the basic model and the clinical model was evaluated (Fig. 3). The AROC increased from 0.640 (95\% CI, 0.609-0.671) in the basic model to 0.648 (95\% CI, 0.617-0.678) in the clinical model. The addition of MAU to the basic model improved the discrimination ability; however, the AROC changed only marginally, from 0.640 to 0.648 (p value 0.099), when MAU was added to the clinical model. 


\begin{tabular}{|l|l|l|l|}
\hline & $\begin{array}{l}\text { Microalbuminuria (+) } \\
(\mathbf{n}=365)\end{array}$ & $\begin{array}{l}\text { Microalbuminuria (-) } \\
(\mathbf{n}=\mathbf{3 0 2 0})\end{array}$ & P value \\
\hline Age (years) & $55 \pm 9$ & $52 \pm 9$ & $<0.001$ \\
\hline Male gender & $125(34.2)$ & $1378(45.6)$ & $<0.001$ \\
\hline Hypertension & $72(19.7)$ & $351(11.6)$ & $<0.001$ \\
\hline Current smoking status & $61(16.7)$ & $675(22.4)$ & 0.069 \\
\hline CAD & $1(0.3)$ & $20(0.7)$ & 0.559 \\
\hline PAD & 0 & $12(0.4)$ & 0.402 \\
\hline Stroke & $5(1.4)$ & $33(1.1)$ & 0.746 \\
\hline SBP, mmHg & $136 \pm 23$ & $123 \pm 19$ & $<0.001$ \\
\hline DBP, mmHg & $87 \pm 13$ & $80 \pm 12$ & $<0.001$ \\
\hline Heart rate & $66 \pm 8$ & $64 \pm 8$ & $<0.001$ \\
\hline Metabolic syndrome & $102(27.9)$ & $817(27.1)$ & 0.709 \\
\hline Triglyceride $\geq 150 \mathrm{mg} / \mathrm{dl}$ & $186(51.0)$ & $1216(40.3)$ & $<0.001$ \\
\hline Low HDL & $214(58.6)$ & $1696(56.2)$ & 0.372 \\
\hline Abdominal obesity & $101(27.7)$ & $911(30.2)$ & 0.334 \\
\hline Hypertension & $60(16.4)$ & $492(16.3)$ & 0.940 \\
\hline Impaired fasting glucose & $70(19.2)$ & $341(11.3)$ & $<0.001$ \\
\hline Creatinine, mg/dl & $0.8 \pm 0.3$ & $0.8 \pm 0.1$ & 0.189 \\
\hline HbA1 , \% & $5.64 \pm 0.4$ & $5.59 \pm 0.3$ & 0.007 \\
\hline Prediabetes & $181(49.6)$ & $1252(41.5)$ & 0.004 \\
\hline BMI, kg/m ${ }^{2}$ & $24.9 \pm 3.3$ & $24.4 \pm 3.1$ & 0.003 \\
\hline Current alcohol & $146(40)$ & $1345(44.5)$ & 0.408 \\
\hline Regular activity & $216(59.2)$ & $1816(60.1)$ & 0.735 \\
\hline Family history of diabetes & $37(10.1)$ & $294(9.7)$ & 0.780 \\
\hline Follow up duration $($ years) & $8.3 \pm 2.8$ & $8.5 \pm 2.6$ & 0.108 \\
\hline
\end{tabular}

Table 1. Baseline characteristics according to the presence of microalbuminuria. Values are presented as mean \pm standard deviation or $\mathrm{n}(\%)$. BMI, body mass index; CAD, coronary artery disease; DBP, diastolic blood pressure; HDL, high-density lipoprotein; HbA1c, haemoglobin Alc; PAD, peripheral artery disease; SBP, systolic blood pressure.

\section{Diabetes-free survival over 10 years}

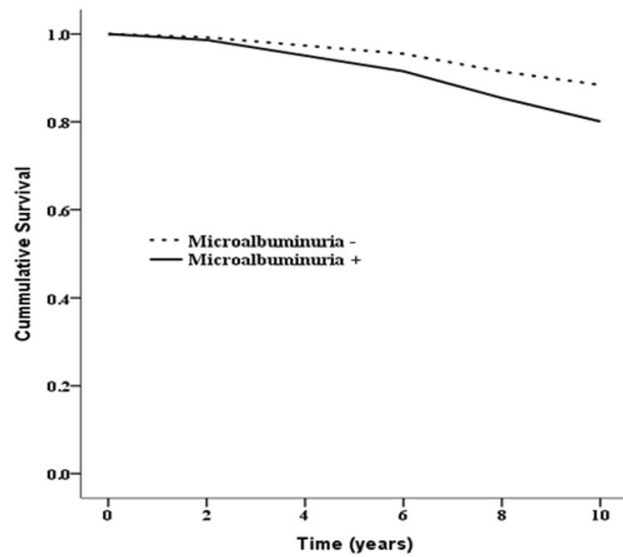

Figure 1. Diabetes free survival curve according to the incident microalbuminuria.

\section{Discussion}

In this large prospective, community-based cohort study of Korean adults, we found that MAU was associated with a 1.43-fold higher risk of development of diabetes after adjusting for several well-known risk factors for diabetes including old age, hypertension, IFG, metabolic component, and family history. The impact of MAU on diabetes was more prominent in subjects without prediabetes.

Several studies have investigated the association of MAU and insulin insensitivity or prediabetes ${ }^{6-8,11}$ Dutta et al. reported that MAU is associated with decreased reversal to normoglycemia and increased progression to diabetes with 147 pre-diabetic individuals with 3 months follow up ${ }^{12}$. Mykkänen et al. reported that MAU is 


\begin{tabular}{|l|l|l|l|}
\hline Clinical event & $\begin{array}{l}\text { Microalbuminuria (+) } \\
(\mathbf{n = 3 6 5})\end{array}$ & $\begin{array}{l}\text { Microalbuminuria (-) } \\
(\mathbf{n = 3 0 2 0})\end{array}$ & P value \\
\hline New diabetes & $56(15.3)$ & $281(9.3)$ & 0.001 \\
\hline Composite event & \multicolumn{2}{|l|}{} \\
\hline CVD & $19(5.2)$ & $138(4.6)$ & 0.597 \\
\hline \multicolumn{5}{|l|}{ Individual events } & $55(1.8)$ & 0.836 \\
\hline Stroke & $7(1.9)$ & $24(0.8)$ & 0.102 \\
\hline Myocardial infarction & 0 & $4(0.1)$ & 0.435 \\
\hline PAD & $1(0.3)$ & $60(2.0)$ & 0.242 \\
\hline CAD & $11(3.0)$ &
\end{tabular}

Table 2. Incidence of diabetes and CVD (stroke, myocardial infarction, CAD, hypertension) in non-diabetic patients during the 10-year follow up. Values are presented as mean \pm standard deviation or $\mathrm{n}(\%)$. CAD, coronary artery disease; CVD, cardio-vascular disease; PAD, peripheral artery disease.

\begin{tabular}{|c|c|c|c|c|}
\hline & \multicolumn{2}{|l|}{ Non-adjusted } & \multicolumn{2}{|l|}{${ }^{\dagger}$ Adjusted } \\
\hline & $\begin{array}{l}\text { Hazard ratio ( } 95 \% \\
\text { confidence interval) }\end{array}$ & P value & $\begin{array}{l}\text { Hazard ratio }(95 \% \\
\text { confidence interval) }\end{array}$ & P value \\
\hline \multicolumn{5}{|l|}{ Overall population } \\
\hline Age $\geq 65$ & $1.47(1.10-2.0)$ & 0.010 & $1.16(0.86-1.55)$ & 0.339 \\
\hline Hypertension & $1.98(1.59-2.47)$ & $<0.001$ & $2.87(0.77-10.70)$ & 0.116 \\
\hline Prediabetes & $8.53(6.88-10.58)$ & $<0.001$ & $5.98(4.47-8.01)$ & $<0.001$ \\
\hline Abdominal obesity & $3.61(2.91-4.48)$ & $<0.001$ & $2.28(1.79-2.90)$ & $<0.001$ \\
\hline Current smoking & $1.41(1.12-1.78)$ & 0.004 & $1.54(1.21-1.95)$ & $<0.001$ \\
\hline Family history of diabetes & $1.45(1.06-1.97)$ & 0.020 & $1.58(1.15-2.16)$ & 0.004 \\
\hline $\mathrm{BMI} \geq 25$ & $2.07(1.67-2.57)$ & $<0.001$ & $1.26(1.00-1.60)$ & 0.049 \\
\hline Microalbuminuria & $1.78(1.34-2.37)$ & $<0.001$ & $1.43(1.07-1.91)$ & 0.016 \\
\hline Regular activity & $1.03(0.83-1.29)$ & 0.770 & & \\
\hline Non-prediabetic population & $\begin{array}{l}\text { Hazard ratio }(95 \% \\
\text { confidence interval) }\end{array}$ & Pvalue & $\begin{array}{l}\text { Hazard ratio }(95 \% \\
\text { confidence interval) }\end{array}$ & P value \\
\hline Age $\geq 65$ & $2.19(1.10-4.34)$ & 0.025 & $1.39(0.69-2.82)$ & 0.361 \\
\hline Hypertension & $2.96(1.73-5.06)$ & $<0.001$ & $2.26(1.28-4.00)$ & 0.005 \\
\hline Abdominal obesity & $2.46(1.42-4.25)$ & 0.001 & $2.26(1.26-4.04)$ & 0.006 \\
\hline Current smoking & $1.68(0.95-2.94)$ & 0.073 & $2.12(1.18-3.81)$ & 0.012 \\
\hline Family history of diabetes & $0.92(0.37-2.31)$ & 0.861 & & \\
\hline $\mathrm{BMI} \geq 25$ & $1.04(0.60-1.81)$ & 0.892 & & \\
\hline Microalbuminuria & $2.78(1.47-5.28)$ & 0.002 & $2.08(1.07-4.03)$ & 0.031 \\
\hline Regular activity & $0.81(04.7-1.37)$ & 0.425 & & \\
\hline
\end{tabular}

Table 3. Multivariate analysis of the overall prevalence of diabetes and the prevalence of diabetes in nonprediabetic subjects. BMI, body mass index.

Hazard ratio of MAU

for development of new diabetes according to subgroup

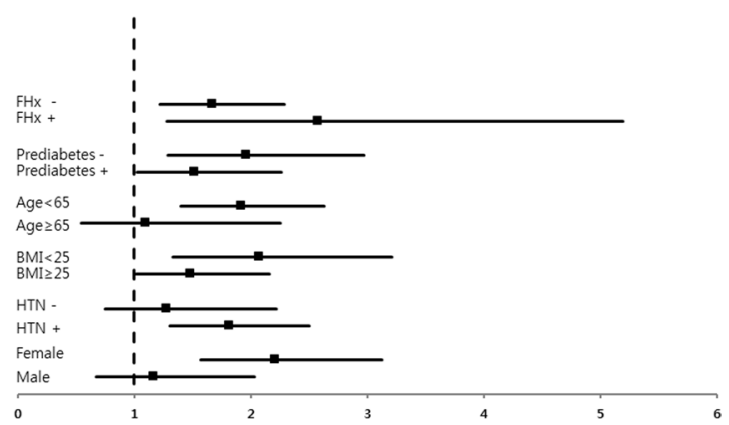

Figure 2. Subgroup analysis for incidental development of diabetes. 


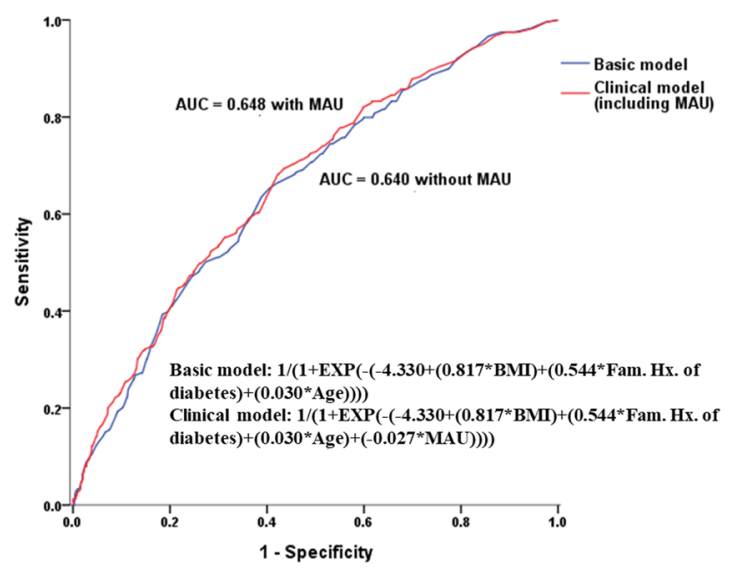

Figure 3. ROC curve for the prediction of diabetes in the basic and the clinical model.

associated with insulin resistance measuring fasting insulin concentration in nondiabetic subjects ${ }^{13}$. However, most of these were cross-sectional studies or small population with short-term follow up data; few data are available regarding whether MAU is directly associated with overt diabetes in large nondiabetic or population-based cohorts.

There is a growing interest in predictors whose abnormal levels indicate an elevated risk of development of diabetes prior to development of clinical symptoms, since regular heath checkups are becoming increasingly common and often include the acquisition of data regarding left ventricular hypertrophy, MAU, high coronary artery calcium score, and increased carotid intima-media thickness. One previous study showed that these subclinical markers are associated with an elevated risk of CVD, especially in people with metabolic syndrome and diabetes ${ }^{11}$. Recently, MAU was recognized as an emerging marker for cardiovascular disease in individuals with hypertension, obese individuals, and the general population, as well as acting as an emerging marker for diabetes ${ }^{14-16}$. The relationship between MAU and insulin insensitivity is based on several pieces of epidemiological evidence, addressing the association of kidney dysfunction with both genetic and non-genetic markers of insulin resistance ${ }^{13,17-19}$. Although many reports have evaluated the relationship between MAU and prediabetes, no clear consensus has yet been reached. In American group, MAU did not predict incident diabetes in pre-diabetic obese subjects $^{20}$. Recently, two cross-sectional studies were performed in Korea. Won et al. ${ }^{21}$ reported that MAU was associated with prediabetes, while Kim et al. concluded that the association is probably mediated by hypertension, and MAU is not independently associated with prediabetes ${ }^{8}$. However, both studies were cross-sectional in nature; thus, they could not show a causal relationship between MAU and prediabetes. In addition, Won et al. did not include HbA1c level in the definition of diabetes, which might have led to an underestimation of the real prevalence of diabetes.

A unique aspect of our study is that we showed a direct association between MAU and incidence of diabetes using a large, nationwide cohort with a mean follow up duration of 8 years. After adjusting for traditional risk factors for diabetes including pre-diabetes and components of metabolic syndrome, the association was still significant. Since the impact of MAU was also statistically significant in subjects without prediabetes, we conclude that MAU is potentially an early marker for diabetes, similar to pre-diabetes. Although the precise mechanism of progression to the diabetes in subjects with MAU is not understood, several epidemiologic and experimental studies have reported potential mechanisms by which insulin resistance is linked to kidney dysfunction in both diabetic and non-diabetic subjects. For instance, there were epidemiologic studies showed that MAU may precede the onset of diabetes ${ }^{12,22}$ in non-diabetic patients. In addition, insulin insensitivity observed in non-diabetic subjects with $\mathrm{MAU}^{13}$ causes podocyte apoptosis and detachment from the glomerular basement membrane ${ }^{23}$ and insulin insensitivity also plays an important role in the development of albuminuria due to endothelial dysfunction and increased vascular permeability, even in non-diabetic patients ${ }^{24}$.

Although we included a large number of participants extracted by stratified random sampling with proportional allocation among the 1476 categories and tried to adjust for confounding factors, this study had some limitations. First, it was difficult to replicate our findings due to lack of availability of another cohort. Thus, lack of replication of our result is one of the major limitations. Second, urinary albumin was assessed in only a single urine specimen per participant. Since urinary albumin level can exhibit considerable intra-individual variability, the lack of repeated sampling could have missed this variation and is thus a limitation. However, there were several studies to evaluate the diagnostic performance of spot urine for the detection of MAU, since 24-hour urine collection is time and cost consuming. They showed that spot urine was a reliable measure of proteinuria in various subjects with diabetes, prediabetes, chronic kidney disease and rheumatic disease as well as in general population ${ }^{25-31}$. Third, MAU could transiently develop in the presence of inflammation or stress. However, we could not fully exclude such conditions, since these data were derived from a nationwide heath cohort. Therefore, another limitation is that we might have overestimated the prevalence of MAU. Fourth, this study was restricted to only Koreans. Therefore, these results might not be applicable to other ethnic groups.

In conclusion, incident MAU could be an early marker for diabetes, even in the non-prediabetic population. Thus, physicians should closely monitor all subjects and regularly measure HbA1C level. Patients should also be educated about lifestyle modifications including weight control, alcohol cessation, and regular activity. 


\section{References}

1. Choi, H. S., Hong, J. W., Lee, J. H., Noh, J. H. \& Kim, D. J. Association of heart rate with albuminuria in a general adult population: the 2011 Korea National Health and Nutrition Examination Survey. Intern Med J 45, 428-435, https://doi.org/10.1111/imj.12672 (2015).

2. Plan and operation of the Third National Health and Nutrition Examination Survey, 1988-94. Series 1: programs and collection procedures. Vital and health statistics. Ser. 1, Programs and collection procedures, 1-407 (1994).

3. Doi, Y. et al. Association of renal resistive index with target organ damage in essential hypertension. American journal of hypertension 25, 1292-1298, https://doi.org/10.1038/ajh.2012.113 (2012).

4. Hillege, H. L. et al. Urinary albumin excretion predicts cardiovascular and noncardiovascular mortality in general population. Circulation 106, 1777-1782 (2002).

5. Yudkin, J. S., Forrest, R. D. \& Jackson, C. A. Microalbuminuria as predictor of vascular disease in non-diabetic subjects. Islington Diabetes Survey. Lancet (London, England) 2, 530-533 (1988).

6. Park, S. K. et al. A cohort study of incident microalbuminuria in relation to HOMA-IR in Korean men. Clin Chim Acta 446, 111-116, https://doi.org/10.1016/j.cca.2015.03.043 (2015).

7. Pilz, S. et al. Insulin sensitivity and albuminuria: the RISC study. Diabetes Care 37, 1597-1603, https://doi.org/10.2337/dc13-2573 (2014).

8. Kim, C. H. et al. Prediabetes is not independently associated with microalbuminuria in Korean general population: the Korea National Health and Nutrition Examination Survey 2011-2012 (KNHANES V-2,3). Diabetes Res Clin Pract 106, e18-21, https://doi. org/10.1016/j.diabres.2014.09.004 (2014).

9. Cho, N. H. et al. Abnormal liver function test predicts type 2 diabetes: a community-based prospective study. Diabetes Care 30, 2566-2568, doi:dc07-0106 [pii] 10.2337/dc07-0106 (2007).

10. Cho, N. H. et al. High Blood Pressure and Its Association With Incident Diabetes Over 10 Years in the Korean Genome and Epidemiology Study (KoGES). Diabetes Care 38, 1333-1338, https://doi.org/10.2337/dc14-1931 (2015).

11. Xanthakis, V. et al. Relations between subclinical disease markers and type 2 diabetes, metabolic syndrome, and incident cardiovascular disease: the Jackson Heart Study. Diabetes Care 38, 1082-1088, https://doi.org/10.2337/dc14-2460 (2015).

12. Dutta, D., Choudhuri, S., Mondal, S. A., Mukherjee, S. \& Chowdhury, S. Urinary albumin: creatinine ratio predicts prediabetes progression to diabetes and reversal to normoglycemia: role of associated insulin resistance, inflammatory cytokines and low vitamin D. J Diabetes 6, 316-322, https://doi.org/10.1111/1753-0407.12112 (2014).

13. Mykkanen, L. et al. Microalbuminuria is associated with insulin resistance in nondiabetic subjects: the insulin resistance atherosclerosis study. Diabetes 47, 793-800 (1998).

14. Minoo, F., Mahdavi-Mazdeh, M., Abbasi, M. R. \& Sohrabi, S. Impact of the severity of obesity on microalbuminuria in obese normotensive nondiabetic individuals. Journal of renal injury prevention 4, 34-38, https://doi.org/10.12861/jrip.2015.08 (2015).

15. Thoenes, M. et al. Albuminuria: pathophysiology, epidemiology and clinical relevance of an emerging marker for cardiovascular disease. Future cardiology 3, 519-524, https://doi.org/10.2217/14796678.3.5.519 (2007).

16. Matsushita, K. et al. Estimated glomerular filtration rate and albuminuria for prediction of cardiovascular outcomes: a collaborative meta-analysis of individual participant data. The Lancet Diabetes \& Endocrinology 3, 514-525, https://doi.org/10.1016/s22138587(15)00040-6 (2015).

17. De Cosmo, S., Menzaghi, C., Prudente, S. \& Trischitta, V. Role of insulin resistance in kidney dysfunction: insights into the mechanism and epidemiological evidence. Nephrology, dialysis, transplantation: official publication of the European Dialysis and Transplant Association - European Renal Association 28, 29-36, https://doi.org/10.1093/ndt/gfs290 (2013).

18. Doria, A. Genetics of diabetes complications. Current diabetes reports 10, 467-475, https://doi.org/10.1007/s11892-010-0147-x (2010).

19. Yip, J. et al. Insulin resistance in insulin-dependent diabetic patients with microalbuminuria. Lancet (London, England) 342 , 883-887 (1993).

20. Friedman, A. N. et al. Value of urinary albumin-to-creatinine ratio as a predictor of type 2 diabetes in pre-diabetic individuals. Diabetes Care 31, 2344-2348, https://doi.org/10.2337/dc08-0148 (2008).

21. Won, J. C. et al. Prevalence of and factors associated with albuminuria in the Korean adult population: the 2011 Korea National Health and Nutrition Examination Survey. PLoS One 8, e83273, https://doi.org/10.1371/journal.pone.0083273 (2013).

22. Mykkanen, L., Haffner, S. M., Kuusisto, J., Pyorala, K. \& Laakso, M. Microalbuminuria precedes the development of NIDDM. Diabetes 43, 552-557 (1994).

23. Welsh, G. I. \& Coward, R. J. Podocytes, glucose and insulin. Current opinion in nephrology and hypertension 19, 379-384, https://doi. org/10.1097/MNH.0b013e32833ad5e4 (2010).

24. Groop, P. H., Forsblom, C. \& Thomas, M. C. Mechanisms of disease: Pathway-selective insulin resistance and microvascular complications of diabetes. Nature clinical practice. Endocrinology \& metabolism 1, 100-110, https://doi.org/10.1038/ncpendmet0046 (2005).

25. Nah, E. H., Cho, S., Kim, S. \& Cho, H. I. Comparison of Urine Albumin-to-Creatinine Ratio (ACR) Between ACR Strip Test and Quantitative Test in Prediabetes and Diabetes. Ann Lab Med 37, 28-33, https://doi.org/10.3343/alm.2017.37.1.28 (2017).

26. Hong, D. S. et al. Evaluation of Urinary Indices for Albuminuria and Proteinuria in Patients with Chronic Kidney Disease. Kidney Blood Press Res 41, 258-266, https://doi.org/10.1159/000443429 (2016).

27. Wu, H. Y. et al. Diagnostic performance of random urine samples using albumin concentration vs ratio of albumin to creatinine for microalbuminuria screening in patients with diabetes mellitus: a systematic review and meta-analysis. JAMA Intern Med 174, 1108-1115, https://doi.org/10.1001/jamainternmed.2014.1363 (2014).

28. Cho, M. C. et al. Evaluation of the URiSCAN super cassette ACR semiquantitative urine dipstick for microalbuminuria screening. Journal of clinical laboratory analysis 28, 281-286, https://doi.org/10.1002/jcla.21681 (2014).

29. Choi, I. A., Park, J. K., Lee, E. Y., Song, Y. W. \& Lee, E. B. Random spot urine protein to creatinine ratio is a reliable measure of proteinuria in lupus nephritis in Koreans. Clinical and experimental rheumatology 31, 584-588 (2013).

30. Gansevoort, R. T. et al. The validity of screening based on spot morning urine samples to detect subjects with microalbuminuria in the general population. Kidney international. Supplement, S28-35, https://doi.org/10.1111/j.1523-1755.2005.09408.x (2005).

31. Zelmanovitz, T. et al. The receiver operating characteristics curve in the evaluation of a random urine specimen as a screening test for diabetic nephropathy. Diabetes Care 20, 516-519 (1997).

\section{Acknowledgements}

We thank all staff who conducted the Korean Genome and Epidemiology Study as well as the civilians who participated in this study. Epidemiological data used in this study were obtained from the Korean Genome and Epidemiology Study (KoGES; KBP 2016-0730117) of the Korea Centres for Disease Control and Prevention, Republic of Korea. 


\section{Author Contributions}

Dong-Hyuk Jung: Conception and design, drafting the manuscript. Young-Sup Byun: Data analysis and drafting the manuscript. Yu-Jin Kwon: Data analysis and drafting the manuscript. Gwang-Sil Kim: Conception and design, revising and final approval of final version.

\section{Additional Information}

Competing Interests: The authors declare that they have no competing interests.

Publisher's note: Springer Nature remains neutral with regard to jurisdictional claims in published maps and institutional affiliations.

(c) (i) Open Access This article is licensed under a Creative Commons Attribution 4.0 International License, which permits use, sharing, adaptation, distribution and reproduction in any medium or format, as long as you give appropriate credit to the original author(s) and the source, provide a link to the Creative Commons license, and indicate if changes were made. The images or other third party material in this article are included in the article's Creative Commons license, unless indicated otherwise in a credit line to the material. If material is not included in the article's Creative Commons license and your intended use is not permitted by statutory regulation or exceeds the permitted use, you will need to obtain permission directly from the copyright holder. To view a copy of this license, visit http://creativecommons.org/licenses/by/4.0/.

(C) The Author(s) 2017 